Article

\title{
Perspectives on Natural Philosophy
}

\author{
Stanley N. Salthe ${ }^{1,2}$ \\ 1 Biological Sciences Department, Binghamton University, Binghamton, NY 13902, USA; \\ ssalthe@binghamton.edu; Tel.: +1-607-467-2623 \\ 2 Biology Department, Brooklyn College, City University of New York, New York, NY 11210, USA
}

Received: 6 August 2018; Accepted: 22 August 2018; Published: 28 August 2018

check for updates

\begin{abstract}
This paper presents a viewpoint on natural philosophy focusing on the organization of substance, as well as its changes as invited by the Second Law of thermodynamics. Modes of change are pointed to as definitive of levels of organization; these include physical, chemical, and biological modes of change. Conceptual uses of the subsumptive hierarchy format are employed throughout this paper. Developmental change in dissipative structures is examined in some detail, generating an argument for the use of final causality in studies of natural systems. Considerations of 'internalism' in science are presented along the way.
\end{abstract}

Keywords: compositional hierarchy; development; dissipative structures; final cause; internalism; Second Law of thermodynamics; subsumptive hierarchy

\section{Introduction}

It is once again the task of natural philosophy (the philosophy of nature) to construct a story about the world and our place in it, based on knowledge from the 'natural' sciences. This requires input from any and all of these sciences that might contribute to the overall picture. My approach (a) follows the developmental impulse of Schelling [1] and (b) is derived mainly from physics and biology, which may generate some distortions in the unfolding picture.

Below, I present a general perspective on the material (including the physical) world using knowledge gained in the special sciences. This requires, in my view, concepts related to substance, change, and direction. Substance is complex, existing at different levels of scale at a single locale. Change 'happens', with, minimally, fluctuations. Directionality characterizes change statistically, leading to ever-new situations. I understand it to be entrained by the Second Law of thermodynamics. Substance tends toward change, while some substantial forms - those organized by energy dissipation-may become directionally transformed by sequentially occupying standard stages-i.e., by 'developing'.

Then, one needs a framework for the various findings of the several natural sciences. This necessitates that only the most basic findings of the sciences are taken up and woven into the overview. As well, it requires a format that is capable of uniting various unrelated concepts that are not known to be in direct interaction within a single framework. My own tool for this purpose has been the hierarchy concept-which actually is two logically different concepts [2]. The compositional hierarchy is based on differences in the scale of activities within a single complex system, while the subsumptive hierarchy is based in set theory, with subsetting representing sequential layering, as in time. This traces minimally an individual history, and, for dissipative structures, stages of development.

\section{Five Basic Conceptual Perspectives}

Hierarchy and Levels

The material world is not just complicated, it is also complex in the sense of being embodied at different levels of organization/scale in one locale. The two known logical forms 
of hierarchy-compositional (boxes within boxes) and subsumptive or specification (steps in construction) - are both useful (I think necessary) in order to properly understand activities at any one level of organization.

\section{Thermodynamics}

The law of nature that is overwhelmingly consequential at all levels of scale, from the smallest to the largest, is the Second Law of thermodynamics. In my view, the Second Law entrains all of the activities studied in natural science, and so may be understood as a final cause of the unfolding of all of the rest. A cosmological understanding of the universe that empowers the Second Law philosophically would be any version of the Big Bang involving an expansion of space.

\section{Dissipative Structures}

A consequence of the Second Law that is of prime importance to humans is the generation, given supportive energy gradients, of dissipative structures at many levels of organization or complexity [3]. All actions beyond some of those considered in fundamental physics lead to irreversible processes, and are initiated and/or mediated by dissipative structures, given appropriate supportive energy gradients.

\section{Development}

The general picture of the universal process is internal differentiation, entraining aggregations of unit processes, as well as resulting in increasing complication locally as the universe expands. The dissipative structures generated or activated by available energy gradients each undergo a characteristic developmental trajectory.

\section{Aristotelian Causal Categories}

The fundamental scenario is a field (formal cause) of possibilities (available material causes), where a momentary configuration or fluctuation elicits/enables an action (efficient cause) that releases local events resulting in a global increase in physical entropy (final cause) as well as changed situations locally. It is my view that the Second Law of thermodynamics in an expanding universe instantiates the importance of final causality in science.

\section{A Note On Internalism}

The general perspective of natural science has been, and is, externalist. The scientist almost universally examines a system from (or as if from) the outside, and is not a part of it, as observed. There have been some exceptions (e.g., the physiologist John Scott Haldane-Wikipedia), while some Buddhists claim to practice an internalist 'science'. Internalism is an attempt to understand a system from within, with the inquirer being a part. Internalism in this sense (not in the mode of the 'humanities') ought to be modest in scope, and focused only locally, as things are happening, and would most appropriately be reported in the present progressive tense [4]. Discursive tendencies that have been suggestive of the possibility of an internalist perspective include Maturana and Varela's [5] 'autopoiesis', dialectics, phenomenology, operationalism in physics, second-order cybernetics, the 'emic' approach in anthropology, and aspects of quantum mechanics.

From the quantum perspective, Rossler [6], noting the dependence of quantum phenomena upon human technological observation, proposed 'endophysics' as a framework for organizing an internalist perspective on the world within science. Elaborated in one way, this view suggests that observation defines the observed-which leads me to note as well Wheeler's [7] 'It from bit' gambit, wherein observation creates the observed, and this view too was stimulated by the dependence of quantum phenomena upon observation (measurement). I note that, as a plain fact, cosmology is necessarily an internalist science; perhaps the best model we have of such a discipline! What needs to be considered in that context is Pattee's [8] argument concerning the necessary 'epistemic cut' in science between the observer and the observed. In cosmology, might this be considered to be achieved when one part of the universe interrogates another, seemingly unconnected part? 
Externally, in science generally, we test things rather than creating them, although we often create the observation platforms, and always create the perspectives from which we make our observations. The main philosophical reason for taking the internalist stance in my opinion would be that (leaving aside quantum mechanics) generativity cannot be approached externally. In the externalist context, nothing radically new is produced except by error. Internally, chance would not differ from choice. Internally, dynamical tendencies are continually under construction during the activities of system self-organization - as informed, not only by current configurations, but also, in my view, by final causes (see below). Of importance in the internalist context may be the concept of vagueness, which is difficult to define [9]. It may best be thought of as an ordinal property. Fuzziness [10] is a conceptual step in this direction, but it is clearly an externalist model. Any system during its development moves from being vaguer to becoming more definitely embodied. Internalism has been of occasional concern to me for some time [11], somewhat inconclusively.

\section{Hierarchy}

The two known logical formulations of hierarchy are important with respect to both substance (the compositional hierarchy) and change- - that is free/open or constrained, i.e., history (the subsumptive, or specification, hierarchy).

Thus, scale is important in the compositional hierarchical perspective:

[Larger scale constraints [level of action of interest (focal level) [smaller scale levels providing enabling conditions on the focal level]]].

Otherwise: [boundary conditions [activities of interest [initiating conditions]]]

This, then, is the compositional hierarchy (Hsc) [2,12], which is most commonly, if not always explicitly, used or implied in scientific thinking, as in:

[larger scale framework [action/observations [smaller scale provisions/affordances]]]

The compositional hierarchy can also be used to relate activities occurring at rates differing by order of magnitude. Rates are characteristically much greater in smaller scale actions than in larger scale activities. Thus, a single measurement of microscopic activities taken at a larger scale might register a small-scale entity as being in, say, two places at the same time, as in some quantum level observations. Activities of focal interest, though relying on and modifying lower-scale supports, generally lead to changes within the scale range of the actions themselves, and may occasionally have consequences as well for larger-scale situations, without changing the logical relations of the framework. Note that the larger-scale framework in science studies often or mostly includes experimental arrangements, and these reflect the nature and interests of the observer's community. This impacts the objectivity of the findings in a very general way.

Explicitly taking account of boundary conditions (more generally, context) in models that are not focused upon results that change the context may be rare in science discourse, even though scientific papers often require a 'Materials and Methods' section, and though the idea of boundary conditions is well established and upfront in physics discourse. Although not commonly appreciated, boundary conditions align with the concept of final cause, which will be discussed further below. Francis Bacon restricted final causality to human actions alone. The scientific observer (taken as an agent of the surrounding culture) is in fact the cause of a scientific observation, which is undertaken for some, mostly implicit, social purpose, as mediated by way of support for the studies. Here is where postmodernism (social constructivism) comes in: its position with regard to science is just that scientific observation cannot discover 'The World' as it would be without effects imported during interactions with particular observers. Scientific discoveries are then used to construct Nature, which, then, is actually nature as it relates to human-indeed, Western cultural-needs. This issue was raised implicitly in quantum mechanics, with the Copenhagen Interpretation. Since that proposal, and continuing now, this view has generated several efforts to obviate it, for example, by searching 
for 'realism' in QM. This can be viewed as a reaction against the implications of social constructivism, which are not much 'appreciated' by most scientists.

Then, concerning change, this can be represented as stages using a Specification Hierarchy, (Hsp) [2], as in (note the different brackets):

\{primal or beginning situation \{modifications accrued \{current or final state\}\}\}

delivering a sequential or historical account of stages in the construction of a present condition, and showing-importantly-also the continuing support of the prior/primal lower levels. A well-known example is the use of this formulation in biological systematics, as in, e.g.,

\{living \{animal \{primate \{human\}\}\}

This particular application in biology was meant only to indicate relationships (humans are primates, etc.), which could be represented using the compositional hierarchy format, but I think it is important to realize that this formulation displays change over time as well. Consider for example, a sentence: 'The boy ran up that hill'. Thus:

$\{$ boy $\{$ ran $\{$ there $\}\}\}$

The boy is the focus of attention; then, his actions are recorded; then, the consequence of those actions is noted-in this case, the boy's current location. The boy existed prior to his act of running, which preexisted its eventual direction. Each new subclass intensifies and focuses more fully upon a continuance of the originally designated object, class, or situation.

Then, consider an unfolding, as in biological development [2]. Thus:

\{ovum \{blastula \{gastrula \{neurula \{etc. \{etc. $\}\}\}\}\}\}$

In this case, the conventional labels for a developing individual label the stage of development achieved (e.g., 'gastrula stage') as development proceeds. This representation illustrates how upstream situations can be viewed as nesting downstream ones by way of their continuing to provide an increasingly modified basic framework.

For yet other possibilities, consult Salthe [11]. The number of subclasses is optional, depending upon the amount of detail required. This format could also be used to illustrate pathways of information flow. In that application, we see that this hierarchy branches as well. A currently active application of that kind is information flow in nervous systems [13-15]. Here, one finds 'heterarchical' relations as well, which I take to refer to the situation where several hierarchies (of either kind) are intersecting in common members. We might say that heterarchy refers to a kind of mashup of hierarchies, rather than an alternative to hierarchy.

Summing up on Hsp, it is used to represent precedence, either temporal, logical, or both. So, an increasingly more specified condition unfolds that continues being subsumed by prior conditions. Thus, a gastrula develops out of a blastula only in time, but insofar as a blastula is a necessary precursor to a gastrula, it is also necessary logically (used loosely here) as providing a prerequisite organization. More examples of use of this hierarchy will unfold in the following text.

\section{Development}

Evolution is mere change. As such, it would occur, even at the quantum level, if a prior condition is not fully recovered in time. In cases where prior conditions could be fully recovered, the definition of 'evolution' would become instead some version of 'change unguided by conserved conditions'. Evolution is non-systematic, and it might impact systematicity only if its occurrence permanently alters a situation.

Development is guided or programmed change. This requires a concept of a system. Developmental change is an unfolding of what is already predestined (déroulement), or of what was implicit or immanent in a system. Here, no matter how elaboratly a system might become as viewed from outside, it continues being itself AS a system, regardless of how extensive the changes undergone were. 
All natural coherent dissipative systems develop intrinsically, and also insofar as they change without being destroyed when stimulated by environmental impingements. That is, they will react characteristically. However, they all evolve during their development as well. Their development is subject to becoming known, while their evolution, even during development, defies prediction. Thus, e.g., in bilaterally symmetric organisms, it is common to observe differences in opposite pairs, as in the right and left ear lobes of mammals, despite both being informed by the same genetic information. I note in passing that the current general discursive interest in evolution rather than development is itself of interest socially, considering that developmental constructions might be favored because they could lead to the possibility of some anticipation and control.

A conceptual application of development can be displayed using a subsumption-specification hierarchy (Hsp), as in:

$$
\text { \{physical world --> \{material world --> \{biological world --> \{social world\}\}\}\} }
$$

showing how the earth became progressively more complex over time. The brackets within brackets format indicates, e.g., that, in the case of the biological world, both the material and physical worlds are still present, underlying and supporting the biological. 'Social world' can be included here as a general category inasmuch as forms of sociality have been observed in most kinds of living systems. Importantly, again, this subsetting format shows that lower integrative levels continue operating within higher (more embedded) ones, as underlying supports. ('Lower' and 'higher' as used here is opposite to the usage in set theory.) Thus, this sequence shows that the social world is simultaneously an aspect of the physical world, a part of the material world, and an extension of the biological world.

Then, for all dissipative structures (located in the material world and above in this hierarchy) the process of development occurs, as:

$$
\text { \{immature --> \{mature --> \{senescent --> \{recycled/dissipated\}\}\}\} }
$$

In this case, unlike the previous one, sequential labels replace each other as development proceeds. However, what was achieved in an earlier stage is still present, even though modified and built upon. The individual referred to in all stages is still the same one.

This is the canonical developmental trajectory [16]. Its basic trajectory was first clearly described in a physical sense by Zotin [17].

A general description of the stages of development for dissipative structures is shown in Table 1 [15]:

Table 1. Developmental Stages of Dissipative Structures (from Salthe, 15).

\begin{tabular}{l} 
IMMATURE STAGE \\
\hline Relatively high energy density (per unit mass) throughflow rate \\
Relatively small size and/or gross mattergy throughput \\
Relatively high rate of acquisition of informational constraints, along with (when applicable) a high growth rate \\
Relatively low internal stability (subject is changing fast), but dynamical stability (persistence) is high \\
Relatively high Homeorhetic stability to same-scale perturbations \\
\hline \multicolumn{1}{c}{ MATURE STAGE } \\
\hline Declining energy density flow rate is still sufficient for recovery from perturbations \\
Size and gross throughput is typical for the kind of system \\
Form is definitive for the kind of system \\
Internal stability adequate for system persistence \\
Homeostatic stability to same-scale perturbations adequate for recovery \\
\hline SENESCENT STAGE \\
\hline Energy density flow rate gradually dropping below functional requirements \\
Gross mattergy throughput high, but its increase is decelerating \\
Form increasingly accumulates deforming marks as a result of encounters, as part of continuing individuation \\
Internal stability of system becoming high to the point of inflexibility \\
Homeostatic stability to same-scale perturbations declining \\
\hline
\end{tabular}


As noted above, the specification/subsumption hierarchy

\{physical world \{material world \{biological world \{social world\}\}\}\}

describes stages in the development of the world, as a process of building new levels, leaving each of the earlier stages still in place (even if they themselves have continued to evolve during this sequence) as supporting presences. The 'lower' (more primal) are still supporting the emergent 'higher' levels. Each level brings in new constraints and opportunities. What each of these levels of specification brings in (or has added) to the universe can be listed as:

the Physical organizational level (historically, the physical developmental stage of the world): Space expansion, gravity, action, waves, rarefaction/thermalization (this becoming prominent upon the expansion of space), initiating the pull of the Second Law of thermodynamics. It might be argued that this initiates time as well, as for example in the interpretation of Annila and Salthe. [18]

the Chemical (material) organizational level or stage: strong force --> matter, electromagnetism, mass action, chemical transformations, clustering, ephemeral individuated locales, fugitive networks/energy gradients, dissipative structures, individual locale duration, local 'ecosystemic' flows.

the Living organizational level or stage: symbolic coding, information, and meaning [8], relatively stable forms --> reproduction, individuated dissipative structures (functional uniqueness, individuality), adaptedness, levels of macroscopic organization locally, traditions/lineages, competitive exclusion, extinction. At this level, time becomes imprinted in nervous systems in ways suggested by Matsuno and Salthe [4].

Thus, as noted above, Hsp records change, whether guided or free:

\{earlier condition \{intermediate condition \{later condition\}\}\}

with new properties appearing with the emergent levels while the prior basis remains in place.

Could this progression continue 'forever', loading in new emergent levels as the universe expands? This would depend upon whether increasingly powerful dissipative systems will be enabled in order to further accelerate the dissipation of such systems, which might grow in size as time continues. At some point, such more powerful systems might destroy more than they create, in effect conspiring with the Second Law to obliterate all form. On that note, I have suggested [19] that dissipative structures were universally required to mediate the Second Law once diffusion was no longer fully effective, after the origin of material objects.

The still recognized relations among the sciences were already appreciated in the late $19^{\text {th }}$ Century by, e.g., Comte, Spencer, and Peirce [16]. This understanding of relations between scientific disciplines can be displayed using a subsumptive hierarchy of disciplines within science.

\{physics \{chemistry \{biology \{sociology \{psychology\}\}\}\}\}

This shows that the prior subject (course contents immediately to the left) establishes a grounding upon which the next inward discourse relies, indicating, for example, that biology might be considered a special kind of chemistry. Some have argued for mathematics to be shown as the earliest stage in this hierarchy, but in my more materialist view, math is taken to be a product of human conceptual advance. Stated otherwise, I see math as an intellectual construct, while I am accepting the scientific view that the fields of scientific inquiry have uncovered (at least some of) Nature's 'true joints'. In this account then, physics is the basis of chemistry, while chemistry is the basis of biology, and so on. Or, looking the other way-top-down rather than bottom-up_-biological systems can be viewed as harnessing chemistry, and physics, to produce and support their forms and activities. This hierarchy shows a tree 
of scientific knowledge with its root in physics, which might be elaborated further in this format to show branches. Thus, there might logically be more than one kind of biology—which could require a redefinition of what biology is-and, as is disturbingly evident around us, we know that there can be more than one kind of social system emergent with the sociological stage.

It is still not clear in my view that quantum mechanics, which some would place at the base of this hierarchy before classical physics, deals with natural physical activities rather than laboratory constructs (taking nature by the throat!). No consequences for the hierarchical tools described here would be impacted by the choice here. However, this statement depends upon another of my views-that notions of QM effects acting directly upon affairs at higher levels of scale must be false. This conclusion arises from my contention that, in the compositional hierarchy as used to model simultaneous activities at different scales, influences can only emanate upward by transformative steps from adjacent levels. Due to the vastly different rates of activity at the different levels, direct interaction is restricted to within one level. Thus, consider the impact that an amount of magnitude 2 would have upon an effect of magnitude 2,000,000. This opinion of mine will likely be challenged.

Logically, in a subsumptive hierarchy, the number of possible coordinate subclasses in the tree increases as we proceed inward. 'Inward' is an important descriptor here. Systems found further within - higher up in —-the hierarchy are subject to more constraints than those located closer to the base of the hierarchy (in physical actions). These constraints are both restrictive and enabling. Thus, animal bodies can run or grow, turn, or swim, but cannot diffuse or-as such while living—oxidize. Then, since we have many more descriptors for biology than for physics, the number of possible meanings (the 'semiotic freedom' of Hoffmeyer [20]) increases in the higher levels of the hierarchy as well.

\section{Causality}

Of particular interest here, I have proposed [21] that the Aristotelian causal categories can often be interrelated in Hsc according to the scale of influence, thus:

[formal causes, final cause [efficient cause [material causes]]]

I have described finality at the different integrative levels in a subsumptive hierarchy \{physicochemical \{biological \{social $\}\}\}$ as \{teleomaty \{teleonomy \{teleology\}\}\} (otherwise: \{physical tendency \{biological function \{intentionality\}\}\} [20,22]), providing a 'teleotalk' lexicon for the different integrative levels.

I believe it is no longer reasonable to ignore final causality in science. Minimally-inasmuch as all of science is carried out by interested parties-finality 'infects' science from the bottom-up (even on Francis Bacon's account!). Science is itself a natural process, and if it discovers a property or aspect of nature, that property is likely to have been present or foreshadowed in the natural world ante-civilization. Of sharpest importance here is my contention [11,23] that the Second Law of thermodynamics represents a final cause in nature, given that the Big Bang involves an expansion of space, continually producing new space into which entropic photons can flow, and which might, in my view, be represented as the universe 'calling for' the production of physical entropy.

\section{Thermodynamics}

Physics studies the basic processes and major tendencies in nature. That being the case, the phenomena that it studies would reasonably be understood to be the grounds from which all else emerges, and must have emerged historically as well. No systems that are susceptible to scientific examination exist that are not either physical neat, or grounded in physical processes. Dynamic natural systems change and in some cases move, utilizing energy to these ends, including during the construction of their own embodiments, and so appropriate available energies are required in order for them to exist. The most fundamental thermodynamic process might (as a model) be taken to be the net creation of free photons from electron-positron couplings (e.g., as in Compton scattering). This process would be mediated by spontaneous irreversible processes at many scales [24]. Irreversible processes have created whatever material things there are. Since everything depends upon (or is) energy flows, 
thermodynamics can be argued to be the most fundamental science, and energetics can be viewed as basic to phenomena at most scales. Thermodynamics has been observed in quantum mechanical constructions as well [25]. It is increasingly being argued by physicists that quantum phenomena underwrite everything else in the hierarchy of nature. That the results of some chemical experiments can be predicted using quantum mechanical principles $[26,27]$ seems to argue for this suggestion.

Thus, the physical realm can reasonably be understood to underlie all systems, from quarks to galaxies, as well as affording the actions necessary for anything to happen at any scale. Energy itself is held in microscopic configurations, in (in some interpretations) bound electrons. As the energy is tapped, these configurations dissipate into energy flows, much of which, because of the low energy efficiency of any effective work [28], produces mostly just free photons-which embody the 'heat energy' not accounted for in the products of work anywhere in nature. These photons flow from regions of higher density of photons toward regions of lower density. This is one view $[29,30]$ of the physical basis of the Second Law of thermodynamics. Irreversible processes are arranged for by the Big Bang as it produces both energy gradients in metastable formations of matter and an ever-increasing space of low energy density that welcomes (or 'calls for') the free photons produced by material interactions. An interesting exception to the generally free escape of photons from matter during energy flows is found in biology, where 'endergonic' chemical reactions capture some energy lost during neighboring 'exergonic' reactions before it escapes into the expanding universe.

Then, there is a philosophically important minority opinion in thermodynamics; the 'maximum entropy production principle' (MEPP), and its derivative, the 'maximum power principle' (MPP) [31]. This relates to the issue of the finality of the Second Law. When an energy gradient is tapped, will the rate at which it becomes depleted proceed as quickly as possible? I think it is reasonable to believe that whatever rate is imposed by a mode of dissipation, it would not be slower than the fastest possible given the constraints. The rate of dissipation would be maximized. Then, it becomes necessary to consider more broadly systems that tap the energy. The fastest possible rate might be explosive, and destructive to particular systems. Dissipative structures that operate in this mode do not survive long (e.g., forest fires, tornados and dust devils). Then it becomes necessary to examine more tenacious dissipative structures, such as organisms, in order to discover how the energy flows are moderated. Even when moderated, we can understand that the flows must be being maximized given the constraints [31]. As organisms age, their energy flows decline, and it is reasonable to suppose that this decline is what entrains the phenomena associated with aging, which in turn provides feedback for further decline. Senescence might be viewed as a negation of an organism's covenant with the Second Law. MPP $[32,33]$ represents the activity levels of what may be referred to as 'delicate systems' - those, like organisms, that could not survive a full MEPP regime. Then, considering the implications of this argument, we could see that we are impelled to be as active as possible on pain of being 'discarded' from the universe- thus, we understand that the Second Law of thermodynamics is a final cause of all growth and activity. Fidgeting is a survival mechanism!

\section{Summary}

The two hierarchy types-Hsc (scale) and Hsp (important with respect to development), and finality are the three key conceptual elements informing my natural philosophy. They reflect form, change, and directionality. I distinguish 'development' from the more general concept 'change' (in which I include 'evolution'). Thus:

\section{\{change\{development\}\}}

Change per se has no form, resulting in occasional (but often, as in biological evolution, important) alterations historically. Development, characteristic of dissipative structures, entails sequential changes leading to senescence and elimination.

The overall conceptual framework assumed in my perspective is the expansion of space in the Big Bang concept. The ongoing 'Bang' gave rise to the emergence of embodiment at increasing 
size scales. Thus, (1) that which is modeled by the compositional hierarchy (Hsc) emerges during Universal expansion, with larger-sized objects being built from smaller ones as a result of gravitation. The rise of (2) dissipative structures at mesoscopic scales accompanies the emergence of energy density gradients as the universe expands so rapidly that matter cannot remain in equilibrium dispersion. These gradients 'materialize' the (3) Second Law of thermodynamics, which previously would have been effected by diffusion alone. Dissipative structures undergo (4) the canonical developmental trajectory, embodying (5) finality, in their entrainment by the global physical goal of universal equilibrium dispersion. This explains why (leaving open how) anything at all happens. The activities of dissipative structures disperse energy gradients, thus serving the finality of the Second Law, while senescence leading to recycling is their own dynamical attractor.

Then, for completeness sake, I note that evolution or individuation occurs at all material scales via unsystematic perturbations. These can give rise to adaptations among the living as mediated by natural selection. Given the way that large scale, or common, environments (oceans, deserts, etc) have a selective influence upon their denizens, they can by way of natural selection generate quasi-systemic effects such as fins, or plant spines, as examples of convergent evolution. In any case, a major result of evolution materially is that all locales have become unique in at least some dimensions.

Living systems bring in new manifestations via the genetic system: information and meaning. Their genetic system manifests an entirely new mode of action in the world: coding, resulting importantly in meaning [8]. Some physicists have opined that codon matching according to the genetic code is physically just another example of correlation. However, a biologist would note that the complex organization of the system that brings about 'correct' matching is where much of the fundamental interest in biological reproduction lies. The complexity of the genetic system, as well as the seeming arbitrariness of the codes, emphasize the difficulty of understanding how this system may have evolved - that is, of understanding the origin of life, which remains an open problem in science. Thus, we have historically a subsumptive hierarchy of natural activities:

\{diffusion \{chemical mass action \{biological coding\}\}\}

with each kind of activity dependent upon the simpler, more widely instantiated activities. Then:

\{change (reflecting the Second Law of thermodynamics) --> \{development --> \{biological genetics: information --> \{meaning \}\}\}\}.

Funding: This research received no external funding.

Acknowledgments: I thank Malcolm Dean, Sungchul Ji, and Addy Pross for providing relevant information. Two reviewers made important suggestions relating to the subsumptive hierarchy.

Conflicts of Interest: The author declares no conflicts of interest.

\section{References}

1. Esposito, J.L. Schelling's Idealism and Philosophy of Nature; Bucknell University Press: Lewisburg, PA, USA, 1997.

2. Salthe, S.N. Hierarchical structures. Axiomathes 2012, 22, 355-383. [CrossRef]

3. Prigogine, I. Introduction to Thermodynamics of Irreversible Processes; Interscience Publishers: New York, NY, USA, 1995.

4. Matsuno, K.; Salthe, S.N. The origin and development of time. Int. J. Gen. Syst. 2002, 31, 377-393. [CrossRef]

5. Maturana, H.R.; Varela, F.J. Autopoiesis and Cognition; D. Reidel Publishing Co.: Dordrecht, The Netherlands, 1980.

6. Rossler, O.E. Endophysics: The World As An Interface; World Scientific Pub Co., Inc.: Singapore, 1998.

7. Wheeler, J.A. Information, physics, quantum: The search for links. In Complexity, Entropy, and the Physics of Information; Zurek, W., Ed.; Addison-Wesley: Redwood City, CA, USA, 1990.

8. Pattee, H.H. The physics of symbols evolved before consciousness. Cosmos Hist. 2015, 11, 269-277.

9. Dietz, R.; Moruzzi, S. Cuts and Clouds: Vagueness, Its Nature, and Logic; Oxford University Press: New York, NY, USA, 2010.

10. Van Deemter, K. Not Exactly: In Praise of Vagueness; Oxford University Press: New York, NY, USA, 2010. 
11. Salthe, S.N. A mode of 'epi thinking' leads to exploring vagueness and finality. In Modes of Explanation: Affordances for Action and Prediction; Lissack, M., Graber, A., Eds.; Palgrave MacMillan: New York, NY, USA, 2014.

12. Salthe, S.N. Evolving Hierarchical Structures: Their Structure and Representation; Columbia University Press: New York, NY, USA, 1985.

13. Botvinic, M.M.; Niv, Y.; Barto, A.C. Hierarchically organized behavior and its neural foundations: A reinforcement learning perspective. Cognition 2009, 113, 262-280. [CrossRef] [PubMed]

14. Friston, K.J. Hierarchical models in the brain. PLoS Comput. Biol. 2008, 4, e1000211. [CrossRef] [PubMed]

15. Vidaurre, D.; Smith, S.M.; Woolrich, M.W. Brain networks dynamics are hierarchically organized in time. Proc. Natl. Acad. Sci. USA 2017, 114, 12827-12832. [CrossRef] [PubMed]

16. Salthe, S.N. Development and Evolution: Complexity and Change in Biology; MIT Press: Cambridge, MA, USA, 1993.

17. Zotin, A.I. Thermodynamic Aspects of Development; Karger AG: Basel, Switzerland, 1972.

18. Annila, A.; Salthe, S.N. Threads of time. ISRN Thermodyn. 2012. Available online: https://www.hindawi. com/journals/isrn/2012/850957/cta/ (accessed on 22 August 2018). [CrossRef]

19. Salthe, S.N. Purpose in nature. Ludus Vitalis 2008, 29, 49-58.

20. Hoffmeyer, J. Biosemiotics: An Examination into the Signs of Life and The Life of Signs; University of Scranton Press: Scranton, PA, USA, 2008.

21. Salthe, S.N. On Aristotle's conception of causality. Gen. Syst. Bull. 2006, 35, 11.

22. Salthe, S.N.; Fuhrman, G. The cosmic bellows: The Big Bang and the Second Law. Cosmos Hist. 2005, 1, $295-318$.

23. Salthe, S.N. Habit-taking, final causation, and the Big Bang theory. In Consensus on Peirce's Concept of Habit; West, D.E., Anderson, M., Eds.; Springer: New York, NY, USA, 2016.

24. Salthe, S.N. The natural philosophy of work. Entropy 2007, 9, 63-99. [CrossRef]

25. Esposito, M. Quantum thermodynamics: A nonequilibrium Green's Function approach. Phys. Rev. Lett. 2015, 114, 080602. [CrossRef] [PubMed]

26. Pople, J.A.; Smith, D.W. Theoretical Models for Chemistry. In Energy Structure and Reactivity, Proceedings of the 1972 Boulder Summer Research Conference on Theoretical Chemistry, Wiley, NY, USA, 26-30 June 1972; John Wiley and Sons Inc.: New York, NY, USA, 1973; pp. 51-61.

27. Mukherjee, S.; Warshel, A. Realistic simulations of the coupling between the protomotive force and the mechanical rotation of the FO-ATPase. Proc. Natl. Acad. Sci. USA 2012, 109, 14876-14881. [CrossRef] [PubMed]

28. Salthe, S.N. Entropy: What does it really mean? Gen. Syst. Bull. 2003, 32, 5-12.

29. Annila, A.; Salthe, S.N. Physical foundations of evolutionary theory. J. Non-Equilib. Thermodyn. 2010, 35, 301-321. [CrossRef]

30. Annila, A.; Salthe, S.N. On intractable tracks. Phys. Essays 2012, 25, 233-238. [CrossRef]

31. Salthe, S.N. Maximum power and maximum entropy production. Finalities in nature. Cosmos Hist. 2010, 6, 114-121.

32. Schneider, E.D.; Sagan, D. Into The Cool; University of Chicago Press: Chicago, IL, USA, 2005.

33. Odum, H.T.; Pinkerton, R.C. Time's speed regulator, the optimum efficiency for maximum output in physical and biological systems. Am. Sci. 1955, 43, 331-343.

(c) 2018 by the author. Licensee MDPI, Basel, Switzerland. This article is an open access article distributed under the terms and conditions of the Creative Commons Attribution (CC BY) license (http://creativecommons.org/licenses/by/4.0/). 